\title{
Performance of 3D PPN against Arbitrarily Maneuvering Target for Homing Phase
}

\author{
Ke-Bo Li, Hyo-Sang Shin, Member IEEE, Antonios Tsourdos, and Min-Jea Tahk, Member IEEE
}

\begin{abstract}
The performance analysis of the three-dimensional (3D) pure proportional navigation (PPN) guidance law was traditionally conducted by considering the cross-coupling effect of two independent two-dimensional (2D) PPN laws in the pitch and yaw planes. This could increase the complexity of the analysis and lead to conservative analysis results, especially when the target has maneuverability. To mitigate this issue, this paper theoretically analyses the performance of 3D PPN directly on a rotating engagement plane using a Lyapunov-like approach. Considering practical issues, the analysis includes not only capturability, but also upper-bounds of heading error, line-of-sight (LOS) rate, commanded acceleration, and closing speed. The analysis results obtained are also demonstrated by using numerical simulation examples. Compared to the previous studies providing the least conservative results, the analysis procedure is significantly simplified and the results are proven to be more practical and less conservative.
\end{abstract}

Index Terms-Homing missile guidance, 3D PPN, Performance analysis, Arbitrarily maneuvering target, Lyapunov-like approach.

\section{INTRODUCTION}

$\mathrm{P}$ ROPORTIONAL Navigation (PN) has been widely accepted for homing missiles due to its robustness and ease of application since its inception in 1940s [1]. The principle of $\mathrm{PN}$ is that the commanded acceleration is proportional to the line of sight (LOS) angular rate and hence the rotation of the LOS is restrained, then the relative status between the missile and target is forced to approach the collision triangle condition.

True Proportional Navigation (TPN) [2], [3] and Pure Proportional Navigation (PPN) [4] [9] are two main PN guidance laws. Commanded acceleration in TPN is normal to LOS, whereas that of PPN is perpendicular to the missile velocity vector. Compared to TPN, it is relatively difficult to deal with the nonlinearity of the engagement dynamics in PPN. This nonlinearity issue made the performance analysis of PPN challenging and led to a few sophisticated mathematical tools

This work was co-supported by the National Natural Science Foundation of China (Grant Nos. 61690210(2017-2021) and 61690213(2017-2021)) and the Hunan Provincial Natural Science Foundation of China (2019JJ50736).

K.-B. Li is with the Department of Applied Mechanics, College of Aerospace Science and Engineering, National University of Defense Technology, Changsha, 410073, China. (e-mail: likeboreal@ nude.edu.cn).

H.-S. Shin, and A. Tsourdos are with the Institute of Aerospace Sciences, SATM, Cranfield University, College Road, Cranfield, Bedfordshire, MK43 OAL, United Kingdom.

M.-J. Tahk is with Department of Aerospace Engineering, KAIST, Daejeon, 305-701 Rep. of Korea. for the analysis. Qualitative methods [4] [7] or linearization methods [8], [9] were natural techniques used to cope with the nonlinearity issue in the performance analysis of PPN. Linearization methods are also used to analyze the performance of TPN, such as [10] [12].

Most of the performance analyses of PPN assumed the speed superiority of the missile over that of the target. Tyan [13], [14] recently investigated the capture region of three-dimensional (3D) PPN against a nonmaneuvering target whose speed is larger than the missile speed. The capture region obtained is of an airfoil shape, which shrinks as the navigation gain of 3D PPN decreases. Tyan [15] further studied the capture region of 3D PPN against a high-speed and nonmaneuvering target under the existence of an observing lag of the LOS rate. Prasanna and Ghose [16] proposed the retro proportional navigation (RPN) in two-dimensional (2D) plane for the purpose of intercepting high speed targets. Ghosh et al. [17] extended RPN in three-dimensional (3D) space and analyzed its capturability against high-speed nonmaneuvering targets. Later, Ghosh et al. [18] proposed composite PN guidance law to achieve the interception of high-speed nonmaneuvering targets with impact angle constraints. These performance analyses of 3D PPN against high speed targets are constrained to the case of nonmaneuvering targets. Recently, Tyan [19], [20] proposed a new approach to examine the capture region, time-to-go, cost, and impact angle of 3D PPN against nonmaneuvering target by using the angle of relative velocity.

Target maneuvers introduce additional nonlinearities to the engagement problem and consequently additional complexity to the performance analysis of PPN. To tackle with the additional complexity, Ha et al. [21] proposed a Lyapunov-like approach and analyzed the performance of PPN against a randomly maneuvering target. Kim et al. [22] later used the Lyapunov-like method to analyze Biased Proportional Navigation (BPN) with a terminal angle constraint. However, these analyses only considered 2D PPN. As target maneuvers generally result in rotation of the engagement plane, the PPN engagement problem against a maneuvering target is most likely to become 3D.

Song and Ha [23] first extended the Lyapunov-like approach to 3D space for the performance analysis of 3D PPN against an arbitrarily maneuvering target. The analysis includes the capturability, intercept time, and maximum overload of 3D PPN, when there is an initial heading error. Since their analysis of 3D PPN was conducted by first considering the pitch and yaw planes separately and then taking the cross-coupling effect 
into account, the content was complex and many redundant variables were involved. Consequently, the conclusions made in their analysis were somewhat conservative. The analysis results in [21] and [23] were under the assumption that the missile speed is larger than that of the target. Moreover, the results showed that, when the target speed is close to the missile speed, the initial heading error should be smaller than a certain value which may become too stringent to be practical. Based on the Lyapunov-like approach, Oh and $\mathrm{Ha}$ [24] proved that, with certain conditions on the navigation gain and the speed ratio between the missile and target, the missile guided by 3D PPN can intercept the target regardless of initial engagement and target acceleration conditions. Later, Oh [25] proved that the capture condition of 2D PPN against arbitrarily maneuvering target is a large-than-one navigation gain, when the missile is with speed advantage. Recently, K. B. Li et al. [26] extended Oh's result to the case of 3D PPN. According to our knowledge, this is the most general result about PPN's capturability thus far. However, in [24] [26], it is not strictly required that the closing speed between the missile and target is always negative. Hence, the required commanded acceleration of PPN to guarantee the capture of target might become extremely large. Besides, an excessive initial heading error or target acceleration may induce a large overload and a long intercept time, which is pragmatically undesirable.

Despite of extensive studies on the performance analysis of PPN, it is evident that there still exist wide discrepancies between the analysis results and the actual performance of 3D PPN. In order to close the discrepancies, this study aims to improve the performance analysis of 3D PPN against an arbitrarily maneuvering target with bounded acceleration. The analysis is conducted under an acceptable initial heading error in the case where the missile has a speed advantage over the target. Since it has been shown that the Lyapunov-like approach is useful in examining the performance of PPN and/or other guidance laws from previous studies [21] [24], the analysis is also based on the Lyapunov-like approach.

This paper utilizes a rotating LOS coordinate system [1], which was also used by Tyan [13] [15], [19], [20], [27] [29] to analyze the performance of PN guidance laws. In Tyan's papers the rotating LOS system is named of LOS fixed coordinate system. However, since it is a moving coordinate system which perfectly shows the rotational principle of the LOS in 3D space, it is called LOS rotation coordinate (LRC) system in this paper. Establishing the kinematic equations in the LRC system relaxes the complexity in the description of the 3D relative motion. It significantly simplifies the analysis process and removes the involvement of redundant variables in the analysis. This analysis framework can also be readily extended to other type of guidance laws [30] [36].

The main contributions of this paper are that:

1) The LRC system is utilized to analyze the $3 D$ relative motion between the missile and the target. The deduced relative kinematic equation becomes quite simple, and the number of involved variables to analyze the performance of 3D PPN is greatly reduced. This makes it possible to obtain less conservative and more general performance analysis results compared with previous literatures.

2) The selection range of the navigation gain of 3D PPN to guarantee the capturability of an arbitrarily maneuvering target is advanced, which is theoretically less conservative than previous results. Furthermore, the selection ranges of the navigation gain of 3D PPN against the arbitrarily maneuvering target to guarantee the boundednesses of 3D LOS angular rate, commanded acceleration, and heading error are presented, respectively. The obtained results are also less conservative than previous results.

3) With the proposed performance analysis results given in this paper, one could properly select the navigation gain of 3D PPN for a missile against an arbitrarily maneuvering target, when the missile maneuverability is limited and the target maneuverability could be previous estimated. Then, the capture of the target could be guaranteed. Besides, the heading error and the commanded acceleration will not exceed the physical limitations of the missile.

The rest of this paper is organized as follows. In Section II, preliminaries including 3D interception geometry, relative kinematics, and research assumptions are addressed. Section III provides the main performance analysis results and compares them to the least conservative results from previous results. Finally, Section V concludes this study after validating the results of the performance analysis by demonstrating results from numerical simulations in Section IV.

\section{PRELIMINARY}

Traditionally, the 3D pursuit is handled by constructing two independent guidance laws in the pitch and yaw planes of the missile and taking their cross-coupling effect into account. This approach may complicate the description of the relative motion due to the cross-coupling effect and introduce some auxiliary variables to the $3 \mathrm{D}$ pursuit problem.

Establishing the kinematic equations in the LRC system could ease the complexity in the description of the 3D relative motion. The relative motion in the LRC system can be divided into two decoupled submotions: 1) the relative motion in the engagement plane spanned by the relative position and velocity vectors and 2) the rotation of this plane. In this paper, these kinematic equations will be used to analyze the performance of 3D PPN.

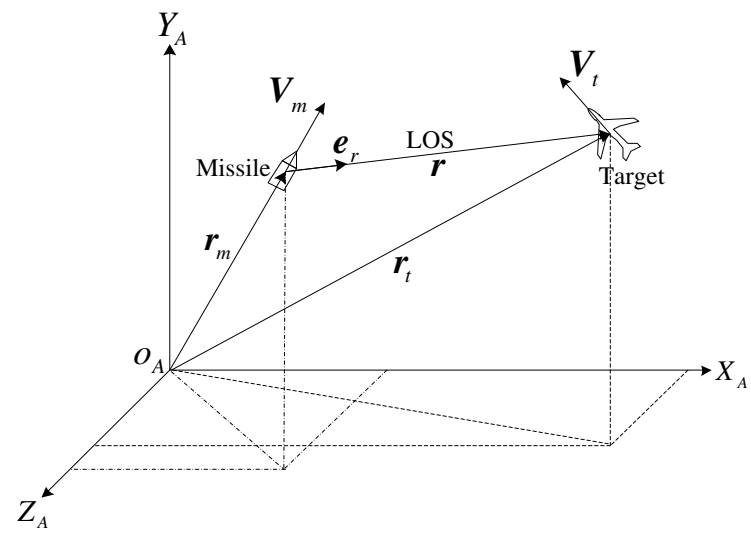

Fig. 1. Three-dimensional engagement geometry. 
This paper considers the 3D engagement problem in which a missile guided by PPN pursues an arbitrarily maneuvering target with time-varying normal acceleration. For simplicity, this paper assumes that:

A1) The missile and target are considered as point masses;

A2) Compared with the resulting overall guidance loop, the autopilot and the seeker dynamics are fast enough to be neglected;

A3) The angle of attack (AOA) and angle of sideslip (AOS) of the missile are small enough to be neglected;

A4) The speeds of missile and target are constant.

It is further assumed that the earth is nonrotating. Note that, these assumptions are generally accepted in the performance analysis of PPN guidance [4] [7], [21] [26].

The 3D pursuit geometry is depicted in Fig. 1. The frame $O_{A}-X_{A} Y_{A} Z_{A}$ denotes the inertial launch frame, which is fixed and centered at the launch site. The origin $O_{A}$ is fixed at the launch point, the $X_{A}$ axis lies in the horizontal plane and points to the launch direction of the missile, while the $Y_{A}$ axis is aligned with the local orthogonal direction of the launch point, and the $Z_{A}$ axis completes a right-handed frame with the other two axes. The position vectors of the missile and target are denoted as $\boldsymbol{r}_{m}$ and $\boldsymbol{r} t$, respectively. The relative position vector $\boldsymbol{r}$ is given by

$$
\boldsymbol{r}=\boldsymbol{r}_{t}-\boldsymbol{r}_{m}
$$

LOS is defined as the direction pointed from the missile to the target, namely,

$$
\boldsymbol{e}_{r}=\boldsymbol{r} / r
$$

where $r$ is the relative range.

Differentiating Eqn. (1) with respect to time gives:

$$
\boldsymbol{v}=\boldsymbol{v}_{t}-\boldsymbol{v}_{m} \Leftrightarrow \dot{\boldsymbol{r}}=\dot{\boldsymbol{r}} \boldsymbol{e}_{r}+\boldsymbol{\omega} \times \boldsymbol{r}=\dot{\boldsymbol{r}} \boldsymbol{e}_{r}+\boldsymbol{\omega}_{s} \times \boldsymbol{r}
$$

where $\boldsymbol{v}_{m}$ and $\boldsymbol{v}_{t}$ denote the velocity vectors of the missile and target, $v$ the relative velocity, i.e., $\dot{\boldsymbol{r}}, \boldsymbol{\omega}$ the angular velocity of $\boldsymbol{r}$, and $\boldsymbol{\omega}_{s}$ the angular velocity of $\boldsymbol{r}$ without the component along $\boldsymbol{e}_{r}$. The plane spanned by $\boldsymbol{r}$ and $\boldsymbol{v}$ is called the engagement plane which is also be called "the instantaneous rotation plane of LOS (IRPL)" [3], [30] [36]. In the analysis, we only consider the case where the missile has speed superiority over the target, i.e., A5)

$$
0<\rho<1
$$

where $\rho:=v_{t} / v_{m}$. Here, $v_{m}$ and $v_{t}$ are the speeds of the missile and target.

The relative velocity in Eqn. (3) can be decomposed into two major components. The first one is the component parallel to LOS and is called radial relative velocity:

$$
\boldsymbol{v}_{r}=v_{r} \boldsymbol{e}_{r}
$$

where $v_{r}=\dot{r}$ denotes the closing speed. The second one is vertical to LOS and is called transversal relative velocity which is given by:

$$
\left\{\begin{array}{l}
\boldsymbol{v}_{\theta}=\boldsymbol{\omega} \times \boldsymbol{r}=\boldsymbol{\omega}_{s} \times \boldsymbol{r}=v_{\theta} \boldsymbol{e}_{\theta} \\
v_{\theta}=r \omega_{s}
\end{array}\right.
$$

where $\boldsymbol{e}_{\theta}$ is the unit vector along $\boldsymbol{\omega}_{s} \times \boldsymbol{r}$, and $\omega_{s}$ is the angular speed of the LOS vector. The angular velocity of LOS, $\omega_{s}$ and its direction $\boldsymbol{e}_{\omega}$ can be represented as:

$$
\boldsymbol{\omega}_{s}=\omega_{s} \boldsymbol{e}_{\omega}, \quad \boldsymbol{e}_{\omega}=\boldsymbol{e}_{r} \times \boldsymbol{e}_{\theta}
$$

LRC is defined as the rotation coordinate frame whose axes are along the unit vectors of $\left(\boldsymbol{e}_{r}, \boldsymbol{e} \theta, \boldsymbol{e}_{\omega}\right) . \boldsymbol{\omega}$ is also the angular velocity of the rotating axes $\left(\boldsymbol{e}_{r}, \boldsymbol{e}_{\theta}, \boldsymbol{e}_{\omega}\right)$, which can be represented as:

$$
\boldsymbol{\omega}=\boldsymbol{\omega}_{s}+\boldsymbol{\Omega}_{s}=\omega_{s} \boldsymbol{e}_{\omega}+\Omega_{s} \boldsymbol{e}_{r}
$$

where $\boldsymbol{\Omega}_{s}$ is the component of $\boldsymbol{\omega}$ along $\boldsymbol{r}$, and $\Omega_{s}$ denotes the angular speed of the engagement plane. Then, follow property holds:

$$
\frac{\mathrm{d}}{\mathrm{d} t}\left[\begin{array}{l}
\boldsymbol{e}_{r} \\
\boldsymbol{e}_{\theta} \\
\boldsymbol{e}_{\omega}
\end{array}\right]=\left[\begin{array}{ccc}
0 & \omega_{s} & 0 \\
-\omega_{s} & 0 & \Omega_{s} \\
0 & -\Omega_{s} & 0
\end{array}\right]\left[\begin{array}{l}
\boldsymbol{e}_{r} \\
\boldsymbol{e}_{\theta} \\
\boldsymbol{e}_{\omega}
\end{array}\right]
$$

Given Eqn. (9), the second time derivative of $\boldsymbol{r}$ is obtained as:

$$
\boldsymbol{a}=: \ddot{\boldsymbol{r}}=\left(\ddot{r}-r \omega_{s}^{2}\right) \boldsymbol{e}_{r}+\left(r \dot{\omega}_{s}+2 \dot{r} \omega_{s}\right) \boldsymbol{e}_{\theta}+r \omega_{s} \Omega_{s} \boldsymbol{e}_{\omega}
$$

where $\boldsymbol{a}$ denotes the relative acceleration vector of the missile with respect to target. The relative kinematic equation set in $\mathrm{LRC}$ is then given by:

$$
\left\{\begin{aligned}
\ddot{r}-r \omega_{s}^{2} & =a_{t r}-a_{m r} \\
r \dot{\omega}_{s}+2 \dot{r} \omega_{s} & =a_{t \theta}-a_{m \theta} \\
r \omega_{s} \Omega_{s} & =a_{t \omega}-a_{m \omega}
\end{aligned}\right.
$$

where $a$ is the magnitude of the acceleration and subscripts $r, \theta$, $\omega$ on variables represent projections of those variables onto the three axes of $\left(\boldsymbol{e}_{r}, \boldsymbol{e} \theta, \boldsymbol{e}_{\omega}\right)$. Variables with subscripts $m$ and $t$ imply those variables of the missile and target.

The first two equations in Eqn. (11) describe the relative motion in IRPL and the third equation represents the rotational principle of IRPL. As shown in Eqn. (11), the first two equations can be decoupled from the third one. For more details, the reader is referred to [1], [3], [26], [30] [36].

PPN guidance law generates acceleration commands in proportion to the LOS rate. In $3 \mathrm{D}$ space, it is expressed as [1]:

$$
\boldsymbol{a}_{\mathrm{PPN}}=N \boldsymbol{\omega}_{s} \times \boldsymbol{v}_{m}
$$

where $N$ is the navigation gain which could be either constant or time-varying. Denoting the direction vector of the missile velocity as $\boldsymbol{t}_{m}$, the commanded acceleration of 3D PPN can be rewritten as:

$$
\boldsymbol{a}_{\mathrm{PPN}}=N v_{m} \omega_{s} \boldsymbol{e}_{\omega} \times \boldsymbol{t}_{m}
$$

Since $\boldsymbol{e}_{\omega} \times \boldsymbol{t}_{m}$ can be expressed as

$$
\boldsymbol{e}_{\omega} \times \boldsymbol{t}_{m}=\left(\boldsymbol{e}_{r} \times \boldsymbol{e}_{\theta}\right) \times \boldsymbol{t}_{m}=-\left(\boldsymbol{t}_{m} \cdot \boldsymbol{e}_{\theta}\right) \boldsymbol{e}_{r}+\left(\boldsymbol{t}_{m} \cdot \boldsymbol{e}_{r}\right) \boldsymbol{e}_{\theta}
$$

it has:

$$
\boldsymbol{a}_{\mathrm{PPN}}=N v_{m} \omega_{s}\left[-\left(\boldsymbol{t}_{m} \cdot \boldsymbol{e}_{\theta}\right) \boldsymbol{e}_{r}+\left(\boldsymbol{t}_{m} \cdot \boldsymbol{e}_{r}\right) \boldsymbol{e}_{\theta}\right]
$$

Note that Eqn. (15) implies that $\boldsymbol{a}$ PPN is always located on IRPL.

It is assumed that the target acceleration is applied perpendicular to its velocity. Then, the target acceleration vector is given by:

$$
\boldsymbol{a}_{t}=a_{t} \boldsymbol{n}_{t}, \quad \boldsymbol{n}_{t} \cdot \boldsymbol{t}_{t}=0
$$

where $\boldsymbol{t}_{t}$ is direction of the target velocity and $\boldsymbol{n}_{t}$ the direction of target acceleration vector which is perpendicular to $\boldsymbol{t}_{t}$. Here, $a_{t}$ is the magnitude of the target acceleration.

For an arbitrarily maneuvering target, $\boldsymbol{n}_{t}$ could be along any direction perpendicular to $\boldsymbol{t}_{t}$, while the magnitude of $\boldsymbol{a}_{t}$ is assumed to be bounded, i.e.:

$$
\left|a_{t}(t)\right| \leq \alpha, \quad t \geq t_{0}=0
$$


where $\alpha(>0)$ denotes the bound of the target acceleration. Throughout the paper, subscript " 0 " on variables means the initial condition of those variables.

The geometric relationship between $\boldsymbol{t}_{m}$ (or $\left.\boldsymbol{t}_{t}\right)$ and $\boldsymbol{e}_{r}$ is shown in Fig. 2.

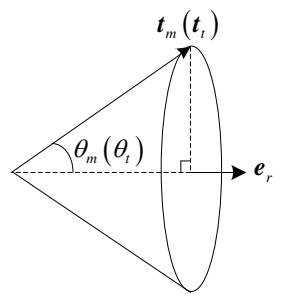

(a) $\theta_{m}\left(\theta_{t}\right) \in[0, \pi / 2]$

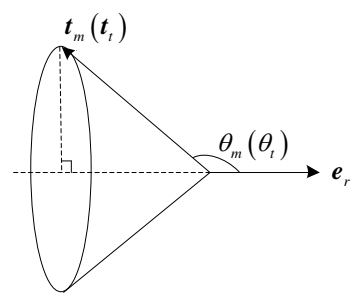

(b) $\theta_{m}\left(\theta_{t}\right) \in(\pi / 2, \pi]$
Fig. 2. Two types of relationships between the directions of missile (target) velocity and LOS.

From Fig. 2, $\theta_{m}$ and $\theta_{t}$ are defined as the angles satisfying the following conditions:

$$
\left\{\begin{array}{l}
\boldsymbol{t}_{m} \cdot \boldsymbol{e}_{r}=\cos \theta_{m} \\
\boldsymbol{t}_{t} \cdot \boldsymbol{e}_{r}=\cos \theta_{t}
\end{array}\right.
$$

If $\cos \theta_{m}>0$, then the missile is flying towards the target. Otherwise, the missile is moving away from the target.

When the missile is flying away from the target, the most important thing is to turn the missile back to make it fly towards to target. This issue had been well discussed in the appendix of Ref. [24] and could be solved by selecting a proper navigation gain. Hence, in this paper the situation is mainly discussed where the missile is initially flying towards the target like in the homing guidance, i.e.,

$$
\boldsymbol{t}_{m 0} \cdot \boldsymbol{e}_{r 0}=\cos \theta_{m 0}>0 \Leftrightarrow \theta_{m 0} \in[0, \pi / 2)
$$

Now, let us define $\boldsymbol{m}$ and $\boldsymbol{t}$ as:

$$
\left\{\begin{array}{c}
\boldsymbol{m}=: \boldsymbol{e}_{r} \times\left(\boldsymbol{t}_{m} \times \boldsymbol{e}_{r}\right)=\boldsymbol{t}_{m}-\left(\boldsymbol{t}_{m} \cdot \boldsymbol{e}_{r}\right) \boldsymbol{e}_{r} \\
\boldsymbol{t}=: \boldsymbol{e}_{r} \times\left(\boldsymbol{t}_{t} \times \boldsymbol{e}_{r}\right)=\boldsymbol{t}_{t}-\left(\boldsymbol{t}_{t} \cdot \boldsymbol{e}_{r}\right) \boldsymbol{e}_{r}
\end{array}\right.
$$

Since, from the definition, $\theta_{m}, \theta_{t} \in[0, \pi]$, it has:

$$
\left\{\begin{array}{c}
|\boldsymbol{m}|=\sin \theta_{m} \\
|\boldsymbol{t}|=\sin \theta_{t}
\end{array}\right.
$$

It can be easily seen that, $\boldsymbol{m}$ and $\boldsymbol{t}$ lie in the plane perpendicular to LOS, which was called "the LOS plane" in Ref. [24]. The angle between $\boldsymbol{m}$ and $\boldsymbol{t}$ is denoted as $\Theta$ which belongs to [0, $\pi]$ in this paper. Then,

$$
\cos \Theta=\frac{\boldsymbol{m} \cdot \boldsymbol{t}}{|\boldsymbol{m}||\boldsymbol{t}|}=\frac{\boldsymbol{t}_{m} \cdot \boldsymbol{t}_{t}-\cos \theta_{m} \cos \theta_{t}}{\sin \theta_{m} \sin \theta_{t}}
$$

From the above definitions and assumptions made in this section, the following kinematics can be obtained:

$$
\begin{gathered}
\dot{r}=\boldsymbol{v} \cdot \boldsymbol{e}_{r}=v_{m}\left(\rho \boldsymbol{t}_{t} \cdot \boldsymbol{e}_{r}-\boldsymbol{t}_{m} \cdot \boldsymbol{e}_{r}\right)=v_{m}\left(\rho \cos \theta_{t}-\cos \theta_{m}\right) \\
v_{\theta}=\boldsymbol{v} \cdot \boldsymbol{e}_{\theta}=v_{m}\left[\rho\left(\boldsymbol{t}_{t} \cdot \boldsymbol{e}_{\theta}\right)-\left(\boldsymbol{t}_{m} \cdot \boldsymbol{e}_{\theta}\right)\right] \\
=v_{m}(\rho \boldsymbol{t}-\boldsymbol{m}) \cdot \boldsymbol{e}_{\theta}=v_{m}|\rho \boldsymbol{t}-\boldsymbol{m}|
\end{gathered}
$$

In the following section, the performance of 3D PPN against the arbitrarily maneuvering target will be deeply analyzed using the Lyapunov-like approach as used in [2], [3], [21] [24],
[33], and [34].

\section{PERFORMANCE ANALYSIS}

When one investigates the performance of 3D PPN, besides capturability, there are a couple of practical constraints that need to be theoretically addressed. The first issue is that, for most of missiles, the field of view (FOV) is restricted in a certain range. If the heading error becomes too large, the physical limitation on FOV might be violated and consequently the missile may lose the track of the target during the engagement. Another issue is that, in some simulation results, if the navigation gain is too small, the commanded acceleration may become too large beyond the physical acceleration limit of the missile. Besides, it is undesirable to have positive closing speed during the engagement. Therefore, this section focuses on investigating not only the capturability, but also characteristics of heading error, LOS rate, commanded acceleration, and closing speed in 3D PPN. Although commonly the navigation gain of PPN, i.e., $N$, is assumed to be constant, however, in this section $N$ is supposed to be time-varying and continuous during the guidance process.

\section{A. Missile Lead Angle}

The following Lemma 1 investigates the range of $\theta_{m}$ in 3D PPN against a maneuvering target, which is very important for the performance analysis of 3D PPN.

Lemma 1: Suppose that the assumptions A1)-A7) hold and the navigation gain $N(t)>1$. Then, the commanded acceleration of $3 D$ PPN represented in Eqn. (13) guarantees $\theta m(t)$ such that:

$$
\theta_{m}(t) \leq \max \left\{\theta_{m 0}, \quad \tan ^{-1}\left(\rho / \sqrt{1-\rho^{2}}\right)\right\}, \quad \forall t \geq t_{0}
$$

Proof: The first time derivative of $\cos \theta_{m}$ defined in Eqn. (18) is obtained as:

$$
\frac{\mathrm{d}\left(\cos \theta_{m}\right)}{\mathrm{d} t}=\dot{\boldsymbol{t}}_{m} \cdot \boldsymbol{e}_{r}+\boldsymbol{t}_{m} \cdot \dot{\boldsymbol{e}}_{r}
$$

Here, $\dot{t}_{m}$ is given by:

$$
\dot{\boldsymbol{t}}_{m}=\frac{\boldsymbol{a}_{m}}{v_{m}}=N \omega_{s}\left[\left(\boldsymbol{t}_{m} \cdot \boldsymbol{e}_{r}\right) \boldsymbol{e}_{\theta}-\left(\boldsymbol{t}_{m} \cdot \boldsymbol{e}_{\theta}\right) \boldsymbol{e}_{r}\right]
$$

and $\dot{\boldsymbol{e}}_{r}=\omega_{s} \boldsymbol{e}_{\theta}$ as defined in the second equation of Eqn. (9). Hence:

$$
\frac{\mathrm{d}\left(\cos \theta_{m}\right)}{\mathrm{d} t}=(1-N) \omega_{s} \boldsymbol{t}_{m} \cdot \boldsymbol{e}_{\theta}
$$

From Eqns. (6) and (24), it has:

$$
\boldsymbol{e}_{\theta}=\frac{v_{m}}{r \omega_{s}}(\rho \boldsymbol{t}-\boldsymbol{m})
$$

Hence:

$$
\begin{aligned}
\boldsymbol{t}_{m} \cdot \boldsymbol{e}_{\theta} & =\frac{v_{m}}{r \omega_{s}} \boldsymbol{t}_{m} \cdot(\rho \boldsymbol{t}-\boldsymbol{m}) \\
& =\frac{v_{m}}{r \omega_{s}} \sin \theta_{m}\left(\rho \sin \theta_{t} \cos \Theta-\sin \theta_{m}\right)
\end{aligned}
$$

Putting Eqn. (30) into Eqn. (28) yield 


$$
\begin{aligned}
\frac{\mathrm{d}\left(\cos \theta_{m}\right)}{\mathrm{d} t} & =(1-N) \omega_{s} \boldsymbol{t}_{m} \cdot \boldsymbol{e}_{\theta} \\
& =\frac{v_{m}}{r}(N-1) \sin \theta_{m}\left(\sin \theta_{m}-\rho \sin \theta_{t} \cos \Theta\right)
\end{aligned}
$$

Since $\theta_{m}, \theta_{t} \in[0, \pi]$ from their definitions in this paper, it is clear that:

$$
\frac{\mathrm{d}\left(\cos \theta_{m}\right)}{\mathrm{d} t} \geq \frac{v_{m}}{r}(N-1) \sin \theta_{m}\left(\sin \theta_{m}-\rho \sin \theta_{t}\right)
$$

Thus, $\dot{\theta}_{m}$ holds the following inequality:

$$
\dot{\theta}_{m} \leq-\frac{v_{m}}{r}(N-1)\left(\sin \theta_{m}-\rho \sin \theta_{t}\right)
$$

Then, the following conditions hold:

$$
\begin{cases}\dot{\theta}_{m}<0, & \text { if } \quad \sin \theta_{m}>\rho \\ \dot{\theta}_{m} \leq 0, & \text { if } \quad \sin \theta_{m}=\rho\end{cases}
$$

Note that $\theta_{m 0} \in[0, \pi / 2)$ from the assumption A7). This implies:

$$
\sin \theta_{m}(t)<\sin \theta_{m 0}, \quad \forall t \geq t_{0}, \quad \text { if } \sin \theta_{m 0}>\rho
$$

Since $\theta_{m}(t)$ is a continuous function of time unless the relative range becomes zero, Eqn. (34) also means that:

$$
\sin \theta_{m}(t) \leq \rho, \quad \forall t \geq t_{0}, \quad \text { if } \quad \sin \theta_{m 0} \leq \rho
$$

Therefore, it has:

$$
\sin \theta_{m}(t) \leq \max \left\{\sin \theta_{m 0}, \rho\right\}, \quad \forall t \geq t_{0}
$$

Together with A7), (37) implies that $\theta_{m}(t) \in[0, \pi / 2)$ for all $t \geq t$. Therefore, the condition in (37) is identical with the following condition:

$$
\cos \theta_{m}(t) \geq \min \left\{\cos \theta_{m 0}, \sqrt{1-\rho^{2}}\right\}, \quad \forall t \geq t_{0}
$$

It is trivial that (37) and (38) lead to (25). Then, this lemma is proven.

Furthermore, according to (34), it is easy to find out that, no matter what value $\theta_{m 0}$ is, $\theta_{m}(t)$ will always enter the range of [0, $\sin -1 \rho$ ], as shown in the following discursion.

Notice that Lemma 1 in this paper is actually a simplified version of [Lemma 1, 24] with less variables.

Discursion 1: Suppose that the assumptions A1)-A7) hold and $N(t)>1$. Then the $3 D P P N$ guidance law guarantees that

$$
\sin \theta_{m}(t) \leq \rho, \quad \forall t \geq t_{c}
$$

where $t_{c}$ is defined as

$$
t_{c}=:\left\{\begin{array}{lll}
0, & \text { if } & \theta_{m}(0) \leq \sin ^{-1} \rho \\
\left\{t>0 \mid \sin \theta_{m}(t)=\rho\right\}, & \text { if } & \theta_{m}(0)>\sin ^{-1} \rho
\end{array}\right.
$$

Proof: Discursion 1 can be easily proven according to (34). $\quad \square$

Remark 1: According to Lemma 1 and Discursion $1, \theta_{m}$ in 3D PPN can be naturally divided into two ranges in the homing phase, i.e., $\theta_{m} \in(\sin -1 \rho, \pi / 2)$ and $\theta_{m} \in[0, \sin -1 \rho]$.

\section{B. Heading Error}

Let us first examine properties of the heading error in 3D PPN. If there is no heading error, i.e. the missile is on the constant-bearing collision course, the transversal relative speed represented in Eqn. (24) is zero. As the missile speed $v_{m}$ is not a control variable, the property of the heading error can be examined by investigating the value of $|\rho t-\boldsymbol{m}|$.

Theorem 1: In addition to A1)-A7), suppose the following two assumptions hold.

A8a) For a constant $\beta \in(0,1+\rho]$, the initial states of the missile and target satisfy the following condition:

$$
\left|\rho \boldsymbol{t}_{0}-\boldsymbol{m}_{0}\right|<\beta
$$

A9a) The navigation gain $N(t)$ is chosen such that:

$$
N(t)>N_{1}(t)=1+\frac{\rho+\alpha r(t) /\left(\beta v_{m}^{2}\right)}{\cos \theta_{m}(t)}, \quad \forall t \geq t_{0}
$$

Then, 3D PPN commanded acceleration defined in Eqn. (12) guarantees that

$$
|\rho \boldsymbol{t}(t)-\boldsymbol{m}(t)|<\beta, \quad \forall t \geq t_{0}
$$

Proof: As $v_{\theta}=v_{m}|\rho t-\boldsymbol{m}|$ according to (24), a Lyapunov-like function $V(t)$ is defined as:

$$
V(t)=\frac{v_{\theta}^{2}(t)}{2}=\frac{\left[r(t) \omega_{s}(t)\right]^{2}}{2}=\frac{v_{m}^{2}[\rho t(t)-\boldsymbol{m}(t)]^{2}}{2}
$$

The first-order derivative of $V(t)$ with respect to time is obtained as:

$$
\dot{V}=r \omega_{s}\left(\dot{r} \omega_{s}+r \dot{\omega}_{s}\right)
$$

From Eqns. (11), (15), and (16), it is clear that:

$$
r \dot{\omega}_{s}+2 \dot{r} \omega_{s}=a_{t} \boldsymbol{n}_{t} \cdot \boldsymbol{e}_{\theta}-N v_{m} \omega_{s} \boldsymbol{t}_{m} \cdot \boldsymbol{e}_{r}
$$

Moreover, from Eqn. (23), $r \dot{\omega}_{s}$ can be written as:

$$
\dot{r} \omega_{s}=v_{m} \omega_{s}\left(\rho \boldsymbol{t}_{t} \cdot \boldsymbol{e}_{r}-\boldsymbol{t}_{m} \cdot \boldsymbol{e}_{r}\right)
$$

Therefore, it has

$$
\dot{r} \omega_{s}+r \dot{\omega}_{s}=a_{t} \boldsymbol{n}_{t} \cdot \boldsymbol{e}_{\theta}-\rho v_{m} \omega_{s} \boldsymbol{t}_{t} \cdot \boldsymbol{e}_{r}+(1-N) v_{m} \omega_{s}\left(\boldsymbol{t}_{m} \cdot \boldsymbol{e}_{r}\right)
$$

Substituting Eqn. (48) into Eqn. (45) yields

$$
\begin{aligned}
\dot{V} & =r \omega_{s}\left[a_{t} \boldsymbol{n}_{t} \cdot \boldsymbol{e}_{\theta}-\rho v_{m} \omega_{s}\left(\boldsymbol{t}_{t} \cdot \boldsymbol{e}_{r}\right)+(1-N) v_{m} \omega_{s}\left(\boldsymbol{t}_{m} \cdot \boldsymbol{e}_{r}\right)\right] \\
& =r v_{m} \omega_{s}^{2}\left[(1-N)\left(\boldsymbol{t}_{m} \cdot \boldsymbol{e}_{r}\right)-\rho\left(\boldsymbol{t}_{t} \cdot \boldsymbol{e}_{r}\right)\right]+r \omega_{s} a_{t} \boldsymbol{n}_{t} \cdot \boldsymbol{e}_{\theta} \\
& \leq r v_{m} \omega_{s}^{2}\left[(1-N) \cos \theta_{m}+\rho\right]+r \omega_{s} a_{t}
\end{aligned}
$$

From the assumptions A6) and A9a), the following inequality holds,

$$
\dot{V}<-\frac{\alpha r^{2} \omega_{s}^{2}}{\beta v_{m}}+\alpha r \omega_{s}=-\frac{2 \alpha V}{\beta v_{m}}+\sqrt{2} \alpha \sqrt{V}
$$

After some algebraic calculations, it has:

$$
\sqrt{V(t)}<\frac{\beta v_{m}}{\sqrt{2}}+\left(\sqrt{V_{0}}-\frac{\beta v_{m}}{\sqrt{2}}\right) \exp \left(-\frac{\alpha}{\beta v_{m}} t\right)
$$

From Eqn. (24) and A8a), it is trivial that:

$$
\sqrt{V_{0}}-\frac{\beta v_{m}}{\sqrt{2}}<0
$$

Hence:

$$
\sqrt{V(t)}<\frac{\beta v_{m}}{\sqrt{2}}, \quad \forall t \geq t_{0}
$$

This proves Eqn. (43) for $t \geq t$.

Remark 2: Theorem 1 implies that, for a missile guided by 3D PPN against an arbitrarily maneuvering target with limited normal acceleration, the heading error can be bounded in a certain range, if the following conditions hold: the initial heading error is acceptable and the navigation gain is chosen 
sufficiently large. Eqn. (42) implies that the bigger the speed ratio $\rho$ is, the larger the navigation gain $N$ is demanded. It can be also noted that the choice of $N(t)$ depends on the target acceleration bound $\alpha$, not on the entire target acceleration profile. Even if Eqn. (41) is not satisfied, according to (51), it could be easily deduced that $V(t)<V_{0}$ and $\sqrt{V(t)}$ will converge to be smaller than $\beta v_{m} / \sqrt{2}$ asymptotically. Therefore, during the homing phase, the target will be limited in the missile seeker's FOV if the navigation gain of 3D PPN is properly chosen according to Eqn. (42).

Note that Ha et al. [21] firstly studied the upper-bound of heading error in 2D PPN by using the Lyapunov-like approach, and later Song and $\mathrm{Ha}$ [23] extended it to 3D space. However, the analysis in [21] was limited to 2D space studies and the analysis results in [23] were too conservative compared with the analysis results in Theorem 1. Besides, in both of [21] and [23], $\beta$ was limited in the range of $(0,1-\rho)$, which was quite stringent when $\rho$ is close to 1 .

\section{LOS Rate and Commanded Acceleration}

Next, let us examine the upper-bound on the magnitude of the commanded acceleration which is a major issue on PPN. Although one could suppose that the commanded acceleration of 3D PPN is diverging as the missile approaches an arbitrarily maneuvering target, the acceleration is actually upper-bounded if the navigation gain $N$ is chosen sufficiently large (but still acceptable). Besides, if $N$ is large enough, the LOS rate $\omega_{s}$ can be also limited in a certain range, which means the upper-bound of the heading error will be gradually decreasing during the engagement. This can be seen in Theorem 2 which will be introduced below.

Theorem 2: In addition to A1)-A7), suppose that the following assumption is satisfied.

A9b) The navigation gain $N(t)$ is chosen such that:

$$
N(t)=N_{2}(t)+\frac{k}{\cos \theta_{m}(t)}, \quad N_{2}(t)=2+\frac{2 \rho}{\cos \theta_{m}(t)}
$$

where $k>0$ is a constant. Then, during the guidance process the $3 D$ LOS rate $\omega_{s}$ satisfies

$$
\omega_{s}(t) \leq \delta=\max \left\{\alpha /\left(v_{m} k\right), \quad \omega_{s 0}\right\}, \quad \forall t \geq t_{0}
$$

and the commanded acceleration of $3 D P P N$ is bounded as:

$$
\left|\boldsymbol{a}_{\mathrm{PPN}}(t)\right| \leq N(t) v_{m} \delta, \quad \forall t \geq t_{0}
$$

Proof: The inequality of Eqn. (55) will be proved by contradiction. If Eqn. (55) does not hold, considering the continuity of $\omega_{s}(t)$, it is trivial that there exists a constant $t_{1}, t_{2}$ $\in\left[t_{0},+\infty\right)$ such that:

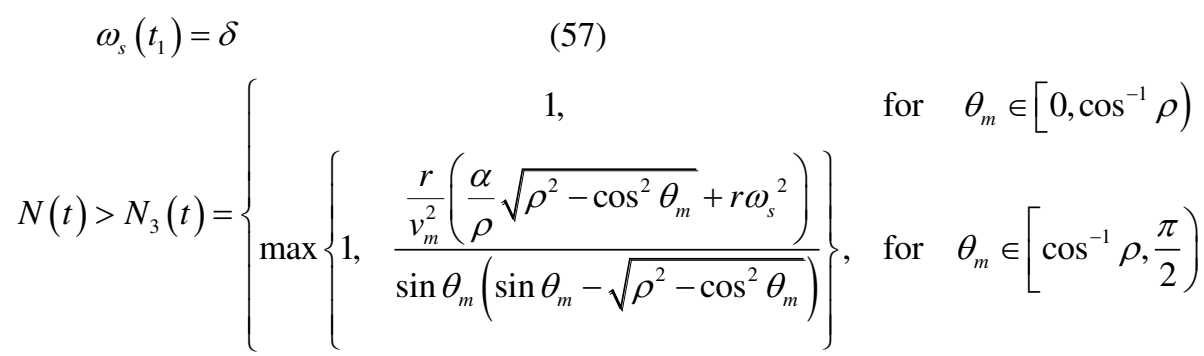

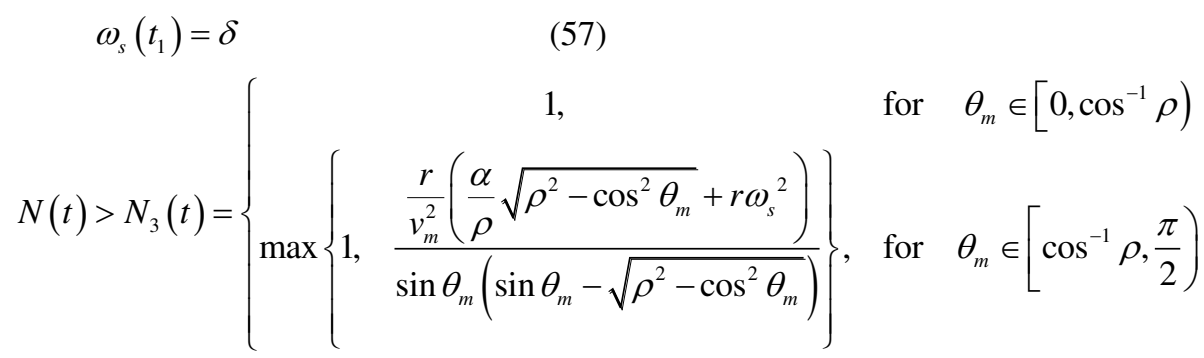

$$
\omega_{s}(t)>\delta, \text { for } t_{1}<t \leq t_{2}
$$

From Eqn. (46), $\dot{\omega}_{s}$ can be obtained as:

$$
\dot{\omega}_{s}=\frac{-2 \dot{r} \omega_{s}+a_{t} \boldsymbol{n}_{t} \cdot \boldsymbol{e}_{\theta}-N v_{m} \omega_{s} \boldsymbol{t}_{m} \cdot \boldsymbol{e}_{r}}{r}
$$

Substituting Eqns. (23) and (54) into Eqn. (59) yields:

$$
\begin{aligned}
\dot{\omega}_{s} & =\frac{(2-N) v_{m} \omega_{s} \cos \theta_{m}-2 v_{m} \omega_{s} \rho \cos \theta_{t}+a_{t} \boldsymbol{n}_{t} \cdot \boldsymbol{e}_{\theta}}{r} \\
& \leq \frac{(2-N) v_{m} \omega_{s} \cos \theta_{m}+2 v_{m} \omega_{s} \rho+\alpha}{r} \\
& \leq-\frac{v_{m} \omega_{s}\left[(N-2) \cos \theta_{m}-2 \rho\right]-\alpha}{r} \\
& =-\frac{\omega_{s} v_{m} k-\alpha}{r}
\end{aligned}
$$

Given the definition of $\delta$ in Eqn. (55) and the bound on $\omega_{s}$ assumed in (58) for $t \in\left(t_{1}, t_{2}\right]$, it is clear that:

$$
\dot{\omega}_{s}<0 \text {, for } t_{1}<t \leq t_{2}
$$

which implies that:

$$
\omega_{s}(t)<\delta \text {, for } t_{1}<t \leq t_{2}
$$

This contradicts Eqn. (58). Hence, Eqn. (55) must hold.

The magnitude of the commanded acceleration in Eqn. (13) is given by:

$$
\left|\boldsymbol{a}_{\mathrm{PPN}}\right|=\left|N v_{m} \omega_{s} \boldsymbol{e}_{\omega} \times \boldsymbol{t}_{m}\right| \leq\left|N v_{m} \omega_{s}\right|
$$

Hence, the validness of (55) leads to the validness of (56).

Remark 3: Measurement errors in the LOS rate result in waste of acceleration energy and even some miss distance. Moreover, the larger the navigation gain is, the worse this becomes. In this aspect, it may not be beneficial to use too large navigation gain. Theorem 2 can give an indication to select the navigation gain in consideration of the overload. If the navigation gain is selected too small, i.e. $k$ is chosen too small, Eqns. (55) and (56) in Theorem 2 indicates that the overload becomes large, probably beyond the physical limitation.

\section{Capturability}

During the engagement, especially at the homing phase, the initial closing speed is usually smaller than 0 and it is desirable to avoid the situation where $\dot{r}$ becomes positive. Moreover, if 3D PPN maintains negative closing speed over the entire engagement, the interception of the target can be guaranteed. Therefore, the following Theorem 3 will investigate the characteristics of the closing speed $\dot{r}$ in 3D PPN.

Theorem 3: In addition to A1)-A7), suppose the following assumption holds.

A9c) $N$ is chosen as: 
Then, 3D PPN of which the commanded acceleration is defined in Eqn. (13) guarantees:

$$
\dot{r}<0, \quad \forall t \geq t_{c}, \text { given } \rho \in(0,1 / \sqrt{2})
$$

where $t_{c}$ is defined as:

$$
t_{c}=: \begin{cases}t_{0}, & \text { if } \sin \theta_{m 0} \leq \rho \\ \left\{t>t_{0} \mid \sin \theta_{m}(t)=\rho\right\}, & \text { if } \sin \theta_{m 0}>\rho\end{cases}
$$

and

$$
\dot{r}<0, \quad \forall t \geq t_{d} \quad \text { given } \quad \rho \in[1 / \sqrt{2}, 1)
$$

where $t_{d}$ is defined as:

$$
t_{d}=: \begin{cases}t_{c}, & \text { if } \dot{r}\left(t_{c}\right)<0 \\ \left\{t>t_{c} \mid \dot{r}(t)=0\right\}, & \text { if } \dot{r}\left(t_{c}\right) \geq 0\end{cases}
$$

Proof. Firstly, Eqn. (65) is proven. From Eqn. (64), $N>1$. Thus, Lemma 1 holds. Therefore, from Lemma 1 and Eqn. (23), if $\sin \theta_{m 0} \leq \rho$, the closing speed meets:

$$
\dot{r} \leq v_{m}\left(\rho-\sqrt{1-\rho^{2}}\right)<0, \quad \forall t \geq t_{0} \quad(\because \rho<1 / \sqrt{2})
$$

If $\sin \theta_{m 0}>\rho$, as shown in Eqn. (34) in the proof of Lemma 1, $\dot{\theta}_{m}$ is negative until $\sin \theta_{m}(t)$ becomes equal to $\rho$. Then, there exists $t_{c}>0$ such that:

$$
\sin \theta_{m}\left(t_{c}\right)=\rho
$$

Note that $t_{c}$ is defined as the time instance when the condition Eqn. (70) is met. From Eqn. (34) in Lemma 1, it has:

$$
\sin \theta_{m}(t) \leq \rho, \quad \forall t \geq t_{c}
$$

Hence, it is obvious that:

$$
\dot{r}(t) \leq v_{m}\left(\rho-\sqrt{1-\rho^{2}}\right), \quad \forall t \geq t_{c}
$$

Since $\rho<1 / \sqrt{2}$, for $\sin \theta_{m 0}>\rho$, Eqn. (65) holds.

Secondly, Eqn. (67) is proven. From Lemma 1, it is clear that Eqn. (71) holds. This implies that

$$
0 \leq \theta_{m} \leq \sin ^{-1} \rho, \quad \forall t \geq t_{c}
$$

We will consider the two regions of $\theta_{m}$ and prove the validness of Eqn. (67) for each region: in the first region $\theta_{m} \in$ $[0, \cos -1 \rho)$, and in the second $\theta_{m} \in[\cos -1 \rho, \pi / 2)$.

If $\theta_{m} \in[0, \cos -1 \rho), \cos \theta_{m}$ is bounded as:

$$
\cos \theta_{m}(t)>\rho, \quad \forall t \geq t_{c}
$$

Then, $\dot{r}(t)$ given in Eqn. (23) is bounded as:

$$
\begin{aligned}
\dot{r} & =v_{m}\left(\rho \cos \theta_{t}-\cos \theta_{m}\right) \quad \forall t \geq t_{d}=t_{c} \\
& <v_{m} \rho\left(\cos \theta_{t}-1\right) \leq 0, \quad
\end{aligned}
$$

Now, let us prove Eqn. (67) for $\theta_{m} \in[\cos -1 \rho, \pi / 2)$. We will first prove Eqn. (67) for the case where $\dot{r}\left(t_{c}\right)<0$ by contradiction.

If the inequality in Eqn. (67) is invalid, for continuous $\dot{r}(t)$ and $\dot{r}\left(t_{c}\right)<0$, there exist $t_{3}, t_{4} \in\left[t_{c},+\infty\right)$ such that:

$$
\begin{gathered}
\dot{r}\left(t_{3}\right)=0 \\
\dot{r}(t)>0, \quad \text { for } t_{3}<t \leq t_{4}
\end{gathered}
$$

From the first equation in Eqn. (11), it has:

$$
\ddot{r}-r \omega_{s}^{2}=a_{t r}-a_{m r}=\boldsymbol{a}_{t} \cdot \boldsymbol{e}_{r}-\boldsymbol{a}_{\mathrm{PPN}} \cdot \boldsymbol{e}_{r}
$$

Substituting Eqns. (15) and (16) into Eqn. (78) yields:

$$
\ddot{r}=a_{t}\left(\boldsymbol{n}_{t} \cdot \boldsymbol{e}_{r}\right)+N v_{m} \omega_{s}\left(\boldsymbol{t}_{m} \cdot \boldsymbol{e}_{\theta}\right)+r \omega_{s}^{2}
$$

From the geometric relationship, it is trivial that

$$
\boldsymbol{n}_{t} \cdot \boldsymbol{e}_{r} \leq \sin \theta_{t}
$$

Given Eqns. (30) and (80), $\ddot{r}$ satisfies the following inequality:

$$
\ddot{r} \leq \alpha \sin \theta_{t}+\frac{N v_{m}^{2}}{r} \sin \theta_{m}\left(\rho \sin \theta_{t}-\sin \theta_{m}\right)+r \omega_{s}^{2}
$$

Since $\dot{r}\left(t_{3}\right)=0, \cos \theta_{m}\left(t_{3}\right)$ meets:

$$
\cos \theta_{m}\left(t_{3}\right)=\rho \cos \theta_{t} \leq \rho
$$

and thus:

$$
\sin \theta_{t}\left(t_{3}\right)=\sqrt{1-\frac{\cos ^{2} \theta_{m}\left(t_{3}\right)}{\rho^{2}}} \geq 0
$$

Therefore, it has:

$$
\begin{aligned}
\sin \theta_{m}\left(t_{3}\right)-\rho \sin \theta_{t}\left(t_{3}\right) & =\sin \theta_{m}\left(t_{3}\right)-\sqrt{\rho^{2}-\cos ^{2} \theta_{m}\left(t_{3}\right)} \\
& =\sqrt{1-\cos ^{2} \theta_{m}\left(t_{3}\right)}-\sqrt{\rho^{2}-\cos ^{2} \theta_{m}\left(t_{3}\right)} \geq 0
\end{aligned}
$$

For the case where $\theta_{m} \in[\cos -1 \rho, \pi / 2), N$ is selected as:

$$
N>\max \left\{1, \frac{\frac{r}{v_{m}^{2}}\left(\frac{\alpha}{\rho} \sqrt{\rho^{2}-\cos ^{2} \theta_{m}}+r \omega_{s}^{2}\right)}{\sin \theta_{m}\left(\sin \theta_{m}-\sqrt{\rho^{2}-\cos ^{2} \theta_{m}}\right)}\right\}
$$

Hence, the inequality in Eqn. (81) at $t=t_{3}$ can be written as:

$$
\begin{aligned}
\ddot{r}\left(t_{3}\right)< & \alpha \sin \theta_{t}\left(t_{3}\right)-\left[\frac{\alpha}{\rho} \sqrt{\rho^{2}-\cos ^{2} \theta_{m}\left(t_{3}\right)}+r\left(t_{3}\right) \omega_{s}^{2}\left(t_{3}\right)\right] \\
& +r\left(t_{3}\right) \omega_{s}^{2}\left(t_{3}\right)
\end{aligned}
$$

Given Eqns. (86) and (83), it is obvious that:

$$
\ddot{r}\left(t_{3}\right)<0
$$

Then, there must exist a time instance $t_{5}$ such that

$$
\dot{r}\left(t_{5}\right)<0, \quad t_{3}<t_{5} \leq t_{4}
$$

which contradicts Eqn. (77).

Now, let us prove Eqn. (67) for $\dot{r}\left(t_{c}\right) \geq 0$, given $\theta_{m} \in[\cos -1 \rho$, $\pi / 2$ ). From Eqn. (23), it is trivial that:

$$
\begin{aligned}
& \dot{r} \geq 0 \quad \quad \cos \theta_{t} \geq \frac{1}{\rho} \cos \theta_{m} \\
& \Leftrightarrow \quad \cos ^{2} \theta_{t} \geq \frac{1}{\rho^{2}} \cos ^{2} \theta_{m} \\
& \quad\left(\because \cos \theta_{m} \text { from Lemma } 1\right) \\
& \Leftrightarrow \quad 1-\cos ^{2} \theta_{t} \leq 1-\frac{1}{\rho^{2}} \cos ^{2} \theta_{m}
\end{aligned}
$$

Hence, for $\dot{r} \geq 0$,

$$
\begin{aligned}
\sin \theta_{m}-\rho \sin \theta_{t} & =\sqrt{1-\cos ^{2} \theta_{m}}-\rho \sqrt{1-\cos ^{2} \theta_{t}} \\
& \geq \sqrt{1-\cos ^{2} \theta_{m}}-\sqrt{\rho^{2}-\cos ^{2} \theta_{m}}>0
\end{aligned}
$$

If $N$ holds the condition in Eqn. (85), from Eqns. (90) and (81), it has $\dot{r} \geq 0$, which is equal to 


$$
\ddot{r} \leq \alpha \sin \theta_{t}+\frac{N v_{m}^{2}}{r} \sin \theta_{m}\left(\rho \sin \theta_{t}-\sin \theta_{m}\right)+r \omega_{s}^{2}<0
$$

This implies that there exists $t_{d}>t_{c}$ such that $\dot{r}\left(t_{d}\right)<0$. Then, it is clear that $\dot{r}$ stays negative for all $t \geq t$. Then, Eqn. (67) is valid. This completes the proof.

Remark 4: According to Theorem 3, a properly chosen navigation gain of 3D PPN will guarantee the capture of the arbitrarily maneuvering target. The smaller the speed ratio $\rho$ is, the smaller the region $\theta_{m} \in[\cos -1 \rho, \pi / 2)$ becomes, where a time-varying $N$ may be needed for guarantee the capturability of 3D PPN. Besides, according to Eqn. (64), a larger upper-bound of the target maneuvering acceleration leads to a larger requirement on $N$ in this region, while the requirement on $N$ reduces, as the relative range decreases.

\section{E. Constant Navigation Gain Design}

Above Lemma 1 and Theorem 1 3 provide a determination method of the navigation gain of 3D PPN, which needs the measurement data of real-time variables of $r(t), \omega_{s}(t)$, and $\theta_{m}(t)$. For radar guided missiles with the inertial measurement unit (IMU), these variables can be measured or estimated during the engagement. Nevertheless, for some types of missiles, it could be desirable to initially determine a proper constant navigation gain which guarantees upper-bounds of heading error, LOS rate, and commanded acceleration and also makes sure negative closing speed during the engagement.

According to Eqns. (42) and (54) in Theorem 1 and 2 in respective, the value of $\theta_{m}(t)$ should not be too big, or the navigation gain must be very large to guarantee the boundednesses of heading error, LOS rate and commanded acceleration of 3D PPN. Therefore, for a homing missile, the initial engagement geometry should be designed to construct a small initial lead angle $\theta_{m}\left(t_{0}\right)$. According to Lemma 1 , if $N>1$ and $\theta_{m}\left(t_{0}\right) \leq \sin -1 \rho, \theta_{m}(t)$ will never be larger than $\sin -1 \rho$ again. Hence, for common missile, it would be reasonable to require that $\theta_{m}\left(t_{0}\right) \leq \sin -1 \rho$ in the homing phase. Besides, for common homing phase, the initial closing speed is often negative.

The following Theorem 4 gives a selection of constant $N$ for 3D PPN to guarantee the boundednesses of heading error, LOS rate, commanded acceleration, and negative closing speed against the arbitrarily maneuvering target in the homing phase.

Theorem 4: For the case where $\dot{r}\left(t_{0}\right)<0$ and $\sin \theta_{m}\left(t_{0}\right) \leq \rho$, suppose A1)-A8a) and the following assumptions hold. A9d) $N$ is chosen such that:

$$
N=2+\frac{2 \rho+k}{\sqrt{1-\rho^{2}}}
$$

where

$$
k>k_{1}=\max \left\{0, \quad \frac{\alpha r_{0}}{\beta v_{m}^{2}}-\left(\sqrt{1-\rho^{2}}+\rho\right)\right\}
$$

for $\rho \in(0,1 / \sqrt{2})$. For $\rho \in[1 / \sqrt{2}, 1)$, $k$ satisfies

$$
k>\max \left\{k_{1},\left[\frac{\frac{\alpha r_{0}}{\rho v_{m}^{2}} \sqrt{2 \rho^{2}-1}+\beta^{2}}{\rho\left(\rho-\sqrt{2 \rho^{2}-1}\right)}-2\right] \sqrt{1-\rho^{2}}-2 \rho\right\}
$$

Then, 3D PPN with the commanded acceleration defined in Eqn. (13) satisfies Eqns. (43), (55), (56), (65) and (67), which imply bounded heading error, bounded 3D LOS rate, bounded commanded acceleration, and negative closing speed, respectively.

Proof: Firstly, Eqns. (55) and (56) are proven to be valid. From the definition of $t_{c}$ in Eqn. (66) and $t_{d}$ in Eqn. (68), for $\dot{r}\left(t_{0}\right)<0$ and $\sin \theta_{m}\left(t_{0}\right) \leq \rho$, it is clear that $t_{c}=t_{d}=t_{0}$.

When $N$ is chosen by (92) and (93), it is clear that $N>1$. According to Lemma 1,

$$
\cos \theta_{m}(t) \geq \sqrt{1-\rho^{2}}, \quad \forall t \geq t_{0}
$$

Then,

$$
N=2+\frac{2 \rho+k}{\sqrt{1-\rho^{2}}} \geq 2+\frac{2 \rho+k}{\cos \theta_{m}(t)}, \quad \forall t \geq t_{0}
$$

Thus, (55) and (56) are valid according to Theorem 2.

Secondly, Eqns. (43), (65) and (67) are proven to be valid. For $\rho \in(0,1 / \sqrt{2})$, according to (92) and (93), it is clear that $N>1$, and hence (65) is valid according to Theorem 3. Besides, recalling (95), the validation of (93) is equal to

$$
N=2+\frac{2 \rho+k}{\sqrt{1-\rho^{2}}}>1+\frac{\rho+\frac{\alpha r(t)}{\beta v_{m}^{2}}}{\cos \theta_{m}(t)}=N_{1}(t), \quad \forall t \geq t_{0}
$$

Hence, according to Theorem 1, (43) is valid during the guidance process.

For $\rho \in[1 / \sqrt{2}, 1)$, assume

$$
r(t) \leq r_{0}, \quad \forall t \geq t_{0}
$$

According to (95) and (98), Eqn. (97) holds, and hence (43) is valid which implies that:

$$
\frac{2 V}{r}=\frac{v_{\theta}^{2}}{r}=r \omega_{s}^{2}<\frac{v_{m}^{2}}{r} \beta, \quad \forall t \geq t_{0}
$$

Note that:

$$
\sin \theta_{m}\left(\sin \theta_{m}-\sqrt{\rho^{2}-\cos ^{2} \theta_{m}}\right)=1-f\left(\cos \theta_{m}\right)
$$

where

$$
f\left(\cos \theta_{m}\right)=\cos ^{2} \theta_{m}+\sqrt{1-\cos ^{2} \theta_{m}} \sqrt{\rho^{2}-\cos ^{2} \theta_{m}}
$$

From Eqn. (84), it is obvious that the Eqn. (100) is positive. According to (74) and (75), we only need to consider the case that $\theta_{m} \in[\cos -1 \rho, \pi / 2)$. In this case, $\cos \theta_{m}$ is bounded as:

$$
\sqrt{1-\rho^{2}} \leq \cos \theta_{m} \leq \rho, \quad \text { for } \rho \in[1 / \sqrt{2}, 1)
$$

Therefore, it is trivial that $f\left(\cos \theta_{m}\right)$ is positive and its derivative with respect to $\cos \theta_{m}$ is negative. We also have:

$$
\rho\left(\rho-\sqrt{2 \rho^{2}-1}\right)=\min \left\{\sin \theta_{m}\left(\sin \theta_{m}-\sqrt{\rho^{2}-\cos ^{2} \theta_{m}}\right)\right\}
$$

and 


$$
\sqrt{1-\rho^{2}}=\underset{\cos \theta_{m}}{\arg \min }\left\{\sin \theta_{m}\left(\sin \theta_{m}-\sqrt{\rho^{2}-\cos ^{2} \theta_{m}}\right)\right\}
$$

Hence, for $\theta_{m} \geq \cos -1 \rho$, recalling (98) it has:

$$
\frac{\frac{r}{v_{m}^{2}}\left(\frac{\alpha}{\rho} \sqrt{\rho^{2}-\cos ^{2} \theta_{m}}+r \omega_{s}^{2}\right)}{\sin \theta_{m}\left(\sin \theta_{m}-\sqrt{\rho^{2}-\cos ^{2} \theta_{m}}\right)} \leq \frac{\frac{\alpha r_{0}}{\rho v_{m}^{2}} \sqrt{2 \rho^{2}-1}+\beta^{2}}{\rho\left(\rho-\sqrt{2 \rho^{2}-1}\right)}
$$

Note that $N$ holding Eqns. (92) (94) in the assumption A9d) satisfies $N>1$. As (94) leads to

$$
N=2+\frac{2 \rho+k}{\sqrt{1-\rho^{2}}}>\frac{\frac{\alpha r_{0}}{\rho v_{m}^{2}} \sqrt{2 \rho^{2}-1}+\beta^{2}}{\rho\left(\rho-\sqrt{2 \rho^{2}-1}\right)}
$$

Combing (105) with (106) leads to (64). Then, according to Theorem 4, it is obvious that (67) hold, which indicates our previous assumption (98) is valid.

Remark 5: According to Theorem 4, if the speed ratio $\rho$, the initial relative range $r$, the upper-bound of the target maneuver acceleration $\alpha$, and the missile speed $v_{m}$ are previously known, a proper constant navigation gain of 3D PPN could be selected, which could guarantee the boundednesses of the heading error, LOS rate, commanded acceleration, and the negative closing speed. Furthermore, if the accurate values of $\rho, r 0, \alpha$, and $v_{m}$ could not be obtained, according to the proof process of Theorem 4, values of $\rho, r 0$, and $\alpha$ could be enlarged a little bit for the selection of $N$, while a smaller value of $v_{m}$ could be adopted.

\section{NUMERICAL SimULATIONS}

In this section, the performance of 3D PPN is demonstrated through numerical simulation. The simulation examples consider the homing guidance phase. The initial conditions are represented in Tab. I.

TABLE 1

Simulation Conditions

\begin{tabular}{l|r|r}
\hline \hline & Parameter & Value \\
\hline Initial missile position & $\boldsymbol{r}_{m 0}$ & $(0,1,0) \mathrm{km}$ \\
Initial relative distance & $r 0$ & $2 \mathrm{~km}$ \\
Initial heading error & $H E_{0}$ & $7.8854 \mathrm{deg}$ \\
Missile speed & $v_{m}$ & $450 \mathrm{~m} / \mathrm{s}$ \\
Target speed & $v t$ & $350 \mathrm{~m} / \mathrm{s}$ \\
Initial LOS elevation angle & $q_{\varepsilon}$ & $30 \mathrm{deg}$ \\
Initial LOS azimuth angle & $q_{\beta 0}$ & $-45 \mathrm{deg}$ \\
Initial missile pitch angle & $\phi_{m}$ & $45 \mathrm{deg}$ \\
Initial missile yaw angle & $\psi_{m}$ & $-45 \mathrm{deg}$ \\
Initial target pitch angle & $\phi_{t}$ & $0 \mathrm{deg}$ \\
Initial target yaw angle & $\psi_{t}$ & $135 \mathrm{deg}$ \\
\hline
\end{tabular}

The sampling rate is $100 \mathrm{~Hz}$. Although the theorems proposed in this paper are valid for any type of maneuvering target whose acceleration meets Eqns. (16) and (17), however, for the length of the paper, we just consider two cases of the arbitrarily maneuvering target where the profiles of target accelerations are dissimilar. The acceleration profile in the first case is given by:

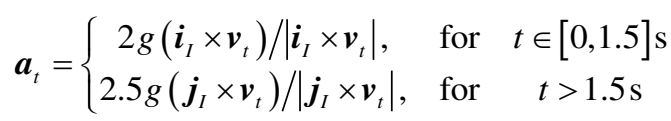

where $\boldsymbol{i}_{I}$ and $\boldsymbol{j}_{\boldsymbol{l}}$ denote the unit vectors corresponding to $x$ and $y$ axes in the inertial coordinate system, and $g$ gravity, i.e., $g \approx$ $9.81 \mathrm{~m} / \mathrm{s} 2$. The second case assumes the following target acceleration profile:

$$
\boldsymbol{a}_{t}=\left\{\begin{array}{ccc}
2 g \sin (2 \pi f t)\left(\boldsymbol{i}_{I} \times \boldsymbol{v}_{t}\right) /\left|\boldsymbol{i}_{I} \times \boldsymbol{v}_{t}\right|, & \text { for } & t \in[0,1.5] \mathrm{s} \\
2.5 g \cos (2 \pi f t)\left(\boldsymbol{j}_{I} \times \boldsymbol{v}_{t}\right) /\left|\boldsymbol{j}_{I} \times \boldsymbol{v}_{t}\right|, & \text { for } & t>1.5 \mathrm{~s}
\end{array}\right.
$$

where $f=1.5 \mathrm{~Hz}$. From the acceleration profile, it has $\alpha=2.5 \mathrm{~g}$. It can be seen that the target maneuvering accelerations considered here are quite complicated for the guidance law to adapt to.

According to Tab. 1 , the initial heading error, $\beta_{0}\left(=: r_{0} \omega_{s 0} / v m\right)$, is approximately equal to 0.1301 and $\rho=0.778>1 / \sqrt{2}$. Note that, according to Section III, $\rho \in[1 / \sqrt{2}, 1)$ is a more stringent region compared with $\rho \in(0,1 / \sqrt{2})$ for the capturability of $3 \mathrm{D}$ PPN. If we set up $\beta=0.2$, Eqn. (92) indicates $N>4.4749$ is large enough to guarantee the capture of the target and the boundednesses of heading error, LOS rate, and commanded acceleration. Thus, for a constant navigation gain, we may choose $N=4.5$.

In this section, it is assumed that $r, \dot{r}, \rho, \omega_{s}$, and $\theta_{m}$ could be accurately measured. Therefore, we could choose the real-time navigation gain $N(t)$ according to Theorem 1 3.

\section{A. Capturability Demonstration}

Firstly, the capturability analysis result of 3D PPN proposed in this paper will be demonstrated and compared with previous results given in [21] and [23]. The analysis methods in [21] and [23] ensure $\dot{r}<0$ in the whole guidance process, while in this paper we ensure $\dot{r}<0$ after $\theta_{m}$ enters [0, sin-1 $\left.\rho\right]$. Note that, according to Discursion $1, \theta_{m}$ will enter $[0, \sin -1 \rho]$ definitely with $N>1$ for 3D PPN. Then, the capture of the target could be guaranteed.

According to Theorem 3, in this case the navigation gain to guarantee $\dot{r}<0$ should be selected as

$$
N=\max \left\{1, \frac{\frac{r}{v_{m}^{2}}\left(\frac{\alpha}{\rho} \sqrt{\rho^{2}-\cos ^{2} \theta_{m}}+r \omega_{s}^{2}\right)}{\sin \theta_{m}\left(\sin \theta_{m}-\sqrt{\rho^{2}-\cos ^{2} \theta_{m}}\right)}\right\}+0.1
$$

while according to [Theorem 1, 21] and [Theorem 1, 23], the navigation gain of PPN should be

$$
N>1+\frac{\rho+\frac{\alpha r_{0}}{\beta v_{m}^{2}}}{\sqrt{1-|\rho+\beta|^{2}}}=\left\{\begin{array}{cc}
10.4870, & \beta=0.2 \\
7.2962, & \beta=0.1301
\end{array}\right.
$$

and

$$
N>1+\frac{\rho+\frac{2 \sqrt{2} \alpha r_{0}}{\beta v_{m}^{2}}}{\sqrt{1-|\rho+\beta|^{2}}}=\left\{\begin{array}{l}
21.0498, \quad \beta=0.2 \\
15.4162, \quad \beta=0.1301
\end{array}\right.
$$


respectively. It can be seen that the analysis result of [Theorem $1,21]$ is more conservative than that in [Theorem 1,23].

The simulation results are shown in Figs. 3 5. During the guidance process, the result of (109) is always $N=1.1$. This is quite smaller than the navigation gain requirements in [21] and [23] to guarantee the capture of an arbitrarily maneuvering target.

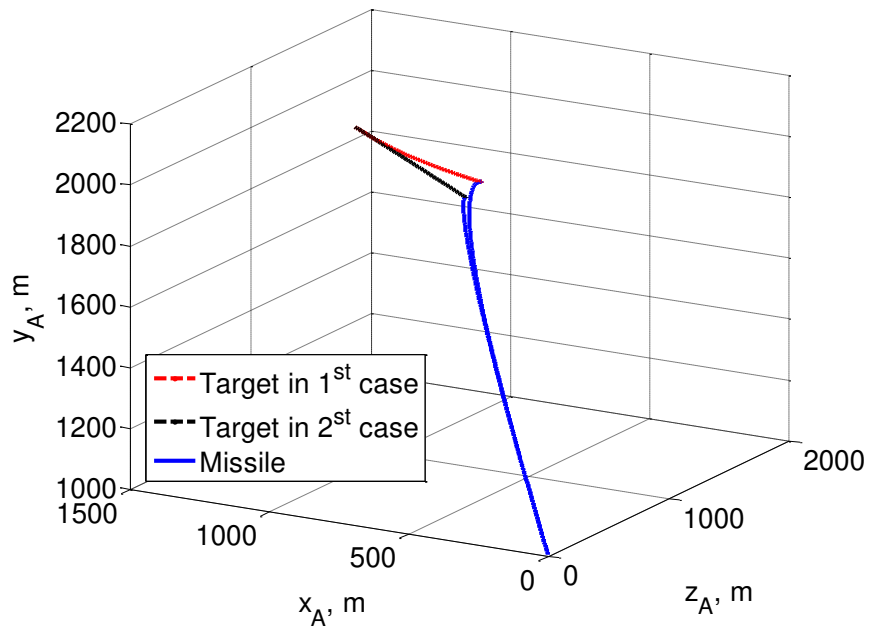

Fig. 3. 3D trajectories of the missile and target (A).

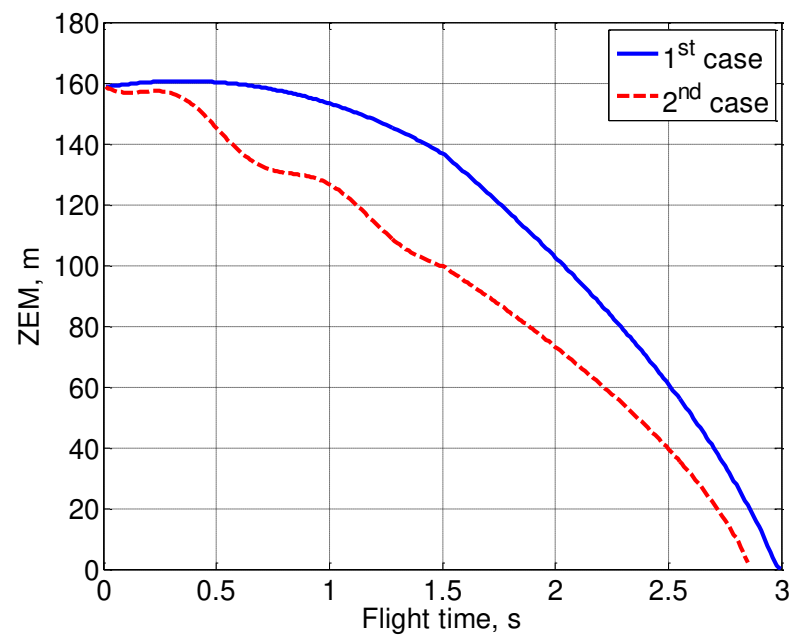

Fig. 4. Zero-effort-miss in two cases (A).

The 3D trajectories and time histories of zero-effort-miss (ZEM) in the two cases are depicted in Fig. 3 and Fig. 4. As shown in these figures, the missile guided by 3D PPN with $N=$ 1.1 successfully intercepts the arbitrarily maneuvering target with bounded acceleration. Fig. 3 illustrates that the two target maneuvers are quite different from each other. Fig. 4 shows that ZEMs are well controlled and converged to zero. The final miss distances in the two cases are smaller than $0.01 \mathrm{~m}$.

The time histories of $\theta_{m}$ and the closing speed $v_{r}$ are illustrated in Fig. 5. According to Lemma 1, for the two cases, as long as $N>1, \theta_{m}(t)$ must be not larger than $\max \left\{22.8854^{\circ}\right.$, $51.0576^{\circ}$, which is valid from the top subfigure of Fig. 5. The closing speed $v_{r}=\dot{r}$ is generally increasing and is always smaller than 0 during the guidance process, which validates the capturability analysis result of this paper, i.e., Theorem 3.
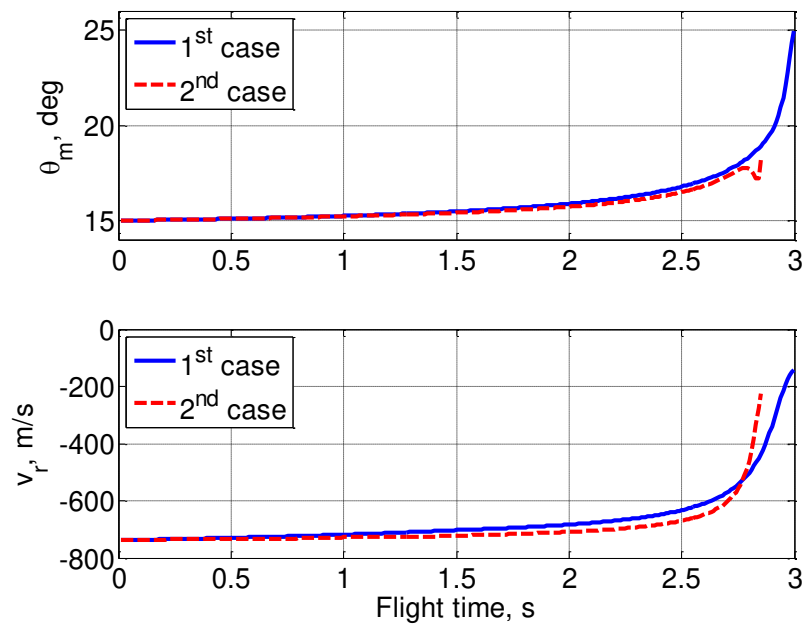

Fig. 5. Time histories of $\cos \theta_{m}$ and the closing speed (A).

\section{B. Upper-Bounds of Heading Error, LOS Rate, and Commanded Acceleration}

Although the above subsection shows that the arbitrarily maneuvering target could be intercepted by 3D PPN with $N$ selected by Eqn. (109), the heading error and commanded acceleration might be extremely large and unacceptable for the realistic seeker and control system of missile. Hence, the navigation gain $N$ should be re-selected by considering the acceptable ranges of heading error, 3D LOS rate, and commanded acceleration, according to Theorem 1 and 2.

In the following content, Theorem 1 and Theorem 2 will be demonstrated.

Firstly, according to Theorem 2, for the limitation of commanded acceleration, the navigation gain is selected as:

$$
N(t)=2+\frac{2 \rho+k}{\cos \theta_{m}(t)}
$$

In this way, (55) and (56) will be valid during the guidance process. Secondly, for the limitation of heading error, (42) needs to be satisfied, hence it has

$$
k>\frac{\alpha r(t)}{\beta v_{m}^{2}}-\cos \theta_{m}(t)-\rho, \quad \forall t \geq t_{0}
$$

Since $\dot{r}(t)<0$ and $\cos \theta_{m}(t) \geq \sqrt{1-\rho^{2}}$ are valid according to Theorem 3 and Lemma 1, respectively, it has

$$
\begin{aligned}
\frac{\alpha r(t)}{\beta v_{m}^{2}}-\cos \theta_{m}(t)-\rho & \leq \frac{\alpha r_{0}}{\beta v_{m}^{2}}-\sqrt{1-\rho^{2}}-\rho \quad \forall t \geq t_{0} \\
& =-0.1952,
\end{aligned}
$$

Hence, a positive $k$ could guarantee the limitation of heading error. Another very important issue is the upper-bound of commanded acceleration. According to Theorem 2, for a positive $k$, the 3D LOS rate $\omega_{s}(t)$ will smaller than $\alpha / v_{m} k$ and the commanded acceleration $\boldsymbol{a} \operatorname{PPN}(t)$ will satisfy

$$
\left|\boldsymbol{a}_{\mathrm{PPN}}(t)\right| \leq \frac{N(t) \alpha}{k} \leq\left[2+\frac{2 \rho+k}{\sqrt{1-\rho^{2}}}\right] \frac{\alpha}{k}
$$

If the upper-bound of the missile maneuverability is assumed as 
$a_{\max }=20 \mathrm{~g}$, then according to Eqn. (112), $k=0.6982$ should be selected. Hence, $k=0.7$ is selected in this subsection.

The simulation results in this case are in Figs. 6 8.

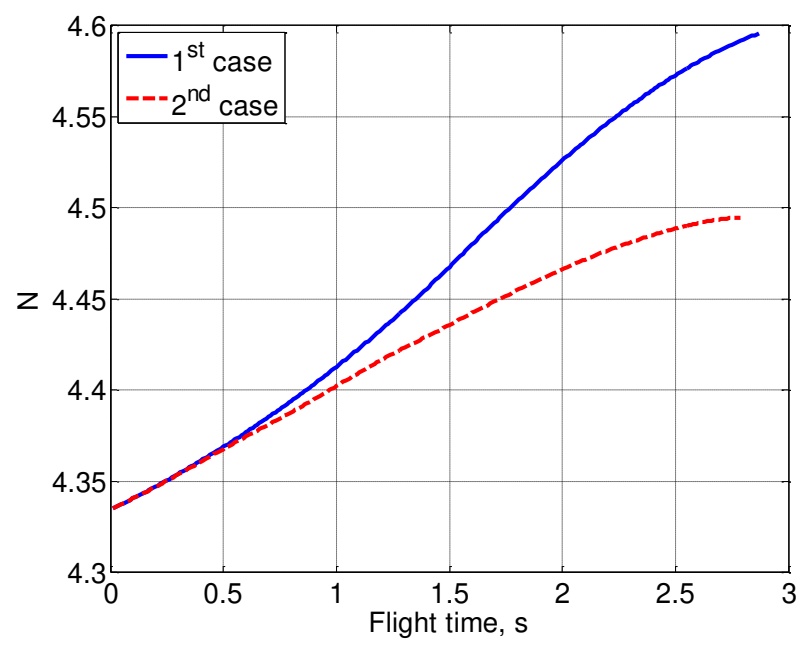

Fig. 6. Navigation gain profiles (B).

Fig. 6 shows the profiles of the navigation gain. In both cases, the navigation gain $N$ is smoothly increasing and is always below 4.6. Since $N$ is much larger than 1.1 here, according to the above subsection, the target can be captured. Hence, the 3D trajectories and the ZEM curves will not be shown in this subsection. The curves of $\theta_{m}$ and $v_{r}$ will also not be shown, as Lemma 1 and Theorem 3 have been demonstrated in the above subsection.

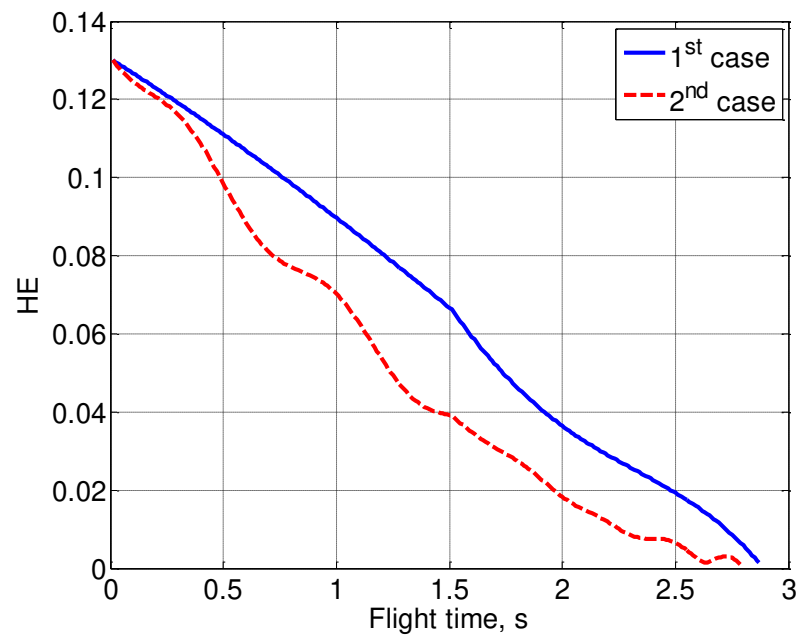

Fig. 7. Heading error profiles (B).

The heading error profiles in both cases are depicted in Fig. 7 . It is clearly shown that the heading error is generally decreasing and converging to zero towards the end of the homing phase. Fig. 7 also shows that the heading error is always smaller than $\beta=0.2$ during the engagement in both cases, which complies with Theorem 1.

The time histories of the total commanded acceleration and the angular speed of the LOS vector are shown in Fig. 8. From Eqn. (55) in Theorem 2, the upper-bound of the 3D LOS rate $\omega_{s}$ would be $\delta=\alpha /(v m k)=4.4609 \mathrm{deg} / \mathrm{s}$, which is strictly valid according to the bottom subfigure of Fig. 8. On the other hand, according to Eqn. (56), the upper-bound of the commanded acceleration would be $\left|\boldsymbol{a}_{\mathrm{PPN}}\right| \leq \max \{N(t)\} v_{m} \delta<4.6 v_{m} \delta=16.4286 \mathrm{~g}$, which is also strictly valid according to the top subfigure of Fig. 8. Besides, from the above analysis, $\left|\boldsymbol{a}_{\mathrm{PPN}}\right| \leq a_{\max }=20 \mathrm{~g}$, which is in accordance with (112). Hence, $k=0.7$ is an appropriate choice for the navigation gain of 3D PPN against the arbitrarily maneuvering target, when $a_{\max }=20 \mathrm{~g}$ under the initial conditions of Tab. 1.
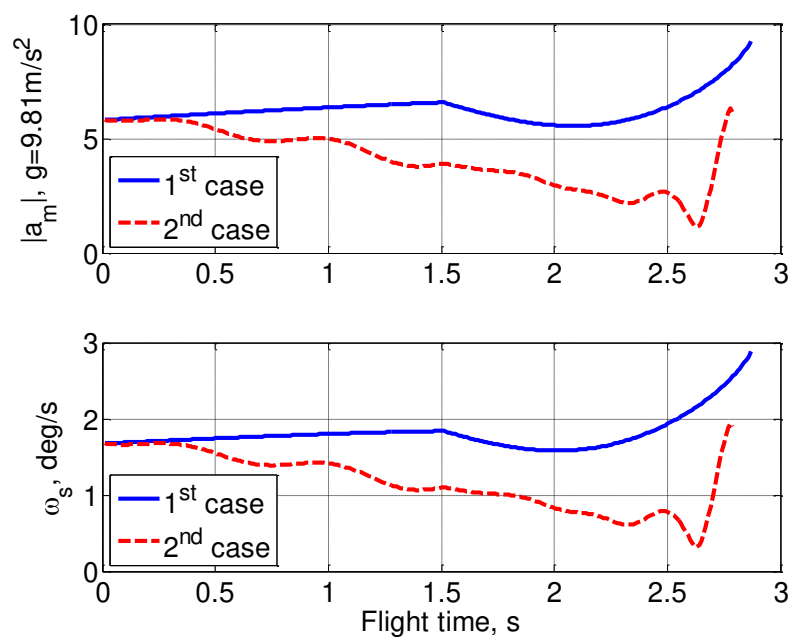

Fig. 8. Time histories of the total commanded acceleration and angular speed of the LOS vector (B).

\section{Conclusions}

This paper analyzed the performance of 3D PPN against an arbitrarily maneuvering target with limited lateral acceleration using a Lyapunov-like method. Unlike previous studies, the analysis in this paper is based on the engagement dynamics established in a rotating coordinated system of LOS to simplify the analysis procedure. From the analysis, it is proven that, if the initial heading error is bounded within a certain range, the 3D PPN guidance law with an acceptable navigation gain guarantees boundedness of the heading error, LOS rate, and commanded acceleration during the pursuit. The analysis results also provide the requirement on the navigation gain to hold the negative closing speed over the engagement. These results were demonstrated using numerical simulations and it is shown that all the simulation results comply with the proposed theories. Compared to previous studies on the performance analysis of 3D PPN, this paper not only significantly simplifies the analysis procedure, but also provides the least conservative results.

One should note that the analysis assumes that the seeker and autopilot have the ideal dynamic characteristics and there is no measurement error. In practice, we should consider the dynamics of the seeker and autopilot, as well as properties of the measurement noise. Nevertheless, the results of the performance analysis in this paper provides a helpful understanding of the characteristics of 3D PPN and the analysis framework can be extended to other PPN-like guidance laws. 


\section{REFERENCES}

[1] N. A. Shneydor, Missile guidance and pursuit: kinematics, dynamics and control. Elsevier, 1998.

[2] K.-B. Li, W.-S. Su, and L. Chen, "Performance analysis of realistic true proportional navigation against maneuvering targets using Lyapunov-like approach," Aerospace Science and Technology, vol. 69 , no. 10 , pp. $333-341$ 2017.

[3] K.-B. Li, Y.-G, Liang, W.-S. Su, and L. Chen, "Performance of 3D TPN against true-arbitrarily maneuvering target for exoatmospheric interception," Sci China Tech Sci, vol. 61, no. 8, pp. 1161 - 1174, 2018.

[4] M. Guelman, "A qualitative study of proportional navigation," Aerospace and Electronic Systems, IEEE Transactions on, vol. 4, no. AES-7, pp. 637 - 643, 1971.

[5] M. Guelman, "Proportional navigation with a maneuvering target," Aerospace and Electronic Systems, IEEE Transactions on, vol. 3, no. AES-8, pp. 364 - 371, 1972.

[6] M. Guelman, "Missile acceleration in proportional navigation," Aerospace and Electronic Systems, IEEE Transactions on, vol.9, no. 3, pp. 462 - 463, 1973.

[7] S. Ghawghawe and D. Ghose, "Pure proportional navigation against time-varying target manoeuvres," Aerospace and Electronic Systems, IEEE Transactions on, vol. 32, no. 4, pp. 1336 - 1347, 1996.

[8] P. Mahapatra and U. Shukla, "Accurate solution of proportional navigation for maneuvering targets," Aerospace and Electronic Systems, IEEE Transactions on, vol. 25, no. 1, pp. 81 - 89, 1989.

[9] U. Shukla and P. Mahapatra, "Generalized linear solution of proportional navigation," Aerospace and Electronic Systems, IEEE Transactions on, vol. 24, no. 3, pp. 231 238, 1988.

[10] P. Zarchan, Tactical and strategic missile guidance, Sixth Edition, AIAA Inc., 2012.

[11] S. Gutman, O. Goldan, and B. Aharon, "Proportional navigation miss distance in the presence of bounded inputs," Journal of Guidance, Control, and Dynamics, vol. 32, no. 4, pp. 1143 - 1151, 2009.

[12] O. Goldan and S. Gutman, "Adjoint stability and miss distance in proportional navigation," Journal of Guidance, Control, and Dynamics, vol. 35, no. 4, pp. 1089 - 1093, 2012.

[13] F. Tyan, "Capture region of 3d PPN guidance law for intercepting high speed target," Joint 48th IEEE Conference on Decision and Control and 28th Chinese Control Conference, Shanghai, P.R. China, Dec. 16-18, 2009, pp. $762-767$.

[14] F. Tyan, "Capture region of a 3d PPN guidance law for intercepting highspeed targets," Asian Journal of Control, vol. 14, no. 5, pp. 1215 - 1226, 2012.

[15] F. Tyan, "Capture region for PPN guidance law with observer lag," in 2011 8th Asian Control Conference (ASCC), 2011.
[16] H. M. Prasanna and D. Ghose, "Retro-proportional-navigation: a new guidance law for interception of high-speed targets, "Journal of Guidance, Control, and Dynamics, vol.35, no. 2, pp. 377 - 386, 2012.

[17] S. Ghosh, D. Ghose, and S. Raha, "Capturability analysis of a 3-d retro-PN guidance law for higher speed nonmaneuvering targets," Control Systems Technology, IEEE Transactions on, vol. 22, no. 5, pp. 1864 - 1874, 2014.

[18] S. Ghosh, D. Ghose, and S. Raha, "Composite guidance for impact angle control against higher speed targets," Journal of Guidance, Control, and Dynamics, vol.39, no. 1, pp. 98 - 117, 2016.

[19] F. Tyan, "Analysis of PPN guidance law-a new approach," in American Control Conference (ACC), 2013. IEEE, 2013, pp. $1030-1035$.

[20] F. Tyan, "Analysis of 3d PPN guidance laws for nonmaneuvering target," Aerospace and Electronic Systems, IEEE Transactions on, vol. 51, no. 4, pp. 2932 2943, 2015.

[21] I.-J. Ha, J.-S. Hur, M.-S. Ko, and T.-L. Song, "Performance analysis of PNG laws for randomly maneuvering targets," Aerospace and Electronic Systems, IEEE Transactions on, vol. 26, no. 5, pp. 713 - 721, 1990.

[22] S. Kim, J. G. Lee, and H. S. Han, "Biased PNG law for impact with angular constraint," Aerospace and Electronic Systems, IEEE Transactions on, vol. 34, no. 1, pp. $277-288,1998$.

[23] S. H. Song and I. J. Ha, "A Lyapunov-like approach to performance analysis of 3-dimensional pure PNG laws," Aerospace and Electronic Systems, IEEE Transactions on, vol. 30, no. 1, pp. 238 - 248, 1994.

[24] J.-H. Oh and I.-J. Ha, "Capturability of the 3-dimensional pure png law," Aerospace and Electronic Systems, IEEE Transactions on, vol. 35, no. 2, pp. 491 - 503, 1999.

[25] J.-H. Oh, "Solving a nonlinear output regulation problem: Zero miss distance of pure PNG," Automatic Control, IEEE Transactions on, vol. 47, no. 1, pp. 169 - 173, 2002.

[26] K.-B. Li, H.-S. Shin, A. Tsourdos, and M.-J. Tahk, "Capturability of 3D PPN against lower-speed maneuvering target for homing phase," Aerospace and Electronic Systems, IEEE Transactions on, available online, vol. 56, no. 1, pp. 771 - 722, 2020.

[27] F. Tyan, "Unified Approach to Missile Guidance Laws: A 3D Extension," Aerospace and Electronic Systems, IEEE Transactions on, vol. 41, no. 4, pp. 1178 - 1199, 2005.

[28] F. Tyan and J. F. Shen, "A Simple Adaptive GIPN Missile Guidance Law," in Proceedings of the 2006 American Control Conference, Minneapolis, Minnesota, USA, June 14-16, 2006, pp. 345-350.

[29] F. Tyan, "Capture region of a GIPN guidance law for missile and target with bounded maneuverability," IEEE Trans. Aerosp. Electron. Syst., vol. 47, no. 1, pp. $201-$ 213, Jan. 2011. 
[30] K.-B. Li, L. Chen, and X.-Z. Bai, "Differential geometric modeling of guidance problem for interceptors," Science China Technological Sciences, vol. 54, no. 9, pp. 2283 2295, 2011.

[31] K.-B. Li, L. Chen, and G. Tang, "Improved differential geometric guidance commands for endoatmospheric interception of high-speed targets," Science China Technological Sciences, vol. 56, no. 2, pp. $518-528$, 2013.

[32] K.-B. Li, L. Chen, and G. Tang, "Algebraic solution of differential geometric guidance command and time delay control," Science China Technological Sciences, vol. 58, no. 3, pp. $565-573,2015$.

[33] K.-B. Li, W.-S., Su, and L. Chen, "Performance Analysis of Three-Dimensional Differential Geometric Guidance Law against low-speed maneuvering targets," Astrodynamics, 2018, 2(3): 233 - 247.

[34] K.-B. Li, W.-S., Su, and L. Chen, "Performance Analysis of Differential Geometric Guidance Law against High-Speed Target with Arbitrarily Maneuvering Acceleration," Proceedings of the Institute of Mechanical Engineerings Part G-Journal of Aerospace Engineering, vol. 233, no. 10, pp. 3547 - 3563, 2019.

[35] H.-S. Shin, A. Tsourdos, and K.-B. Li, "A new three-dimensional sliding mode guidance law variation with finite time convergence," Aerospace and Electronic Systems, IEEE Transactions on, vol. 53, no. 5, pp. 2221 2232, 2017.

[36] K.-B. Li, H.-S. Shin, and A. Tsourdos, "Capturability of a sliding mode guidance law with finite time convergence" Aerospace and Electronic Systems, IEEE Transactions on, available online, Otc. 2019.

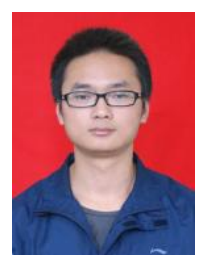

Ke-bo Li received the B.S., M.S., and Ph.D. degrees from the National University of Defense Technology, Changsha, China, in 2008, 2011, and 2016, respectively. Now, he is a Lecturer in Department of Applied Mechanics, College of Aerospace Science and Engineering, National University of Defense Technology. His main research interests include flight vehicle dynamics, guidance, and control.

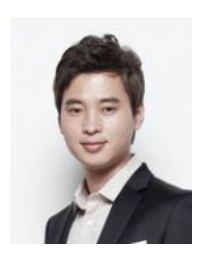

Hyo-Sang Shin received his BSc from Pusan National University in 2004 and gained an MSc on flight dynamics, guidance and control in Aerospace Engineering from KAIST and a $\mathrm{PhD}$ on cooperative missile guidance from Cranfield University in 2006 and 2011, respectively. He is currently a Professor of Guidance, Control and Navigation Systems in Autonomous and Intelligent Systems Group at Cranfield University. His current research interests include multiple target tracking, data-centric guidance and control, and distributed control of multiple agent systems.

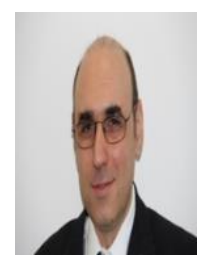

Antonios Tsourdos obtained a MEng in electronic, control and systems engineering from the University of Sheffield (1995), an MSc in systems engineering from Cardiff University (1996), and a $\mathrm{PhD}$ in nonlinear robust missile autopilot design and analysis from Cranfield University (1999). He is a Professor of Control Engineering with Cranfield University, and was appointed Head of the Centre for Autonomous and Cyber-Physical Systems in 2013. He was a member of the Team Stellar, the winning team for the UK MoD Grand Challenge (2008) and the IET Innovation Award (Category Team, 2009).

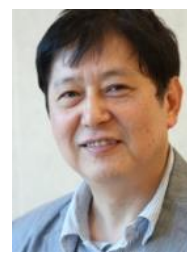

Min-Jea Tahk is Professor of Aerospace Engineering at KAIST (Korea Advanced Institute of Science and Technology). He received the B.S. degree from Seoul National University, Seoul, Korea, and the M.S. and Ph.D. degrees from the University of Texas, Austin, in 1983 and 1986, respectively, all in aerospace engineering. From 1976 to 1981 , he was a research engineer at the Agency for Defense Development in Korea, and from 1986 to 1989 he was employed by Integrated Systems, Inc., Santa Clara, CA, before he joined KAIST. Prof. Tahk was Technical Editor of IEEE Transactions on Aerospace and Electronic Systems from 2011 to 2016. He had also served as Editor-in-Chief of International Journal of Aeronautical and Space Sciences from 2015 to 2018. His recent research interests are missile guidance laws, weapon-target assignment, multiple target tracking, and convex programming for real-time applications.

Fig. 1. Three-dimensional engagement geometry.

Fig. 2. Two types of relationships between the directions of missile (target) velocity and LOS.

Fig. 3. 3D trajectories of the missile and target (A).

Fig. 4. Zero-effort-miss in two cases (A).

Fig. 5. Time histories of $\cos \theta_{m}$ and the closing speed (A).

Fig. 6. Navigation gain profiles (B).

Fig. 7. Heading error profiles (B).

Fig. 8. Time histories of the total commanded acceleration and angular speed of the LOS vector (B). 